\title{
ON THE SPACE OF SUBSETS OF A UNIFORM SPACE
}

\section{A. P. ROBERTSON AND WENDY ROBERTSON ${ }^{1}$}

The set $\varepsilon$ of closed nonempty subsets of a metric space $E$ can be made into a metric space by means of the well-known Hausdorff metric (Hausdorff $[3, \S 28]$ ). When $E$ is complete, so is $\varepsilon$ (see e.g. Kuratowski [6, §29, no. IV]; Price [7]; Bourbaki [1, Chapter IX, $\S 2$, ex. 7]). In a similar way, if $E$ is a uniform space, the uniform structure on $E$ induces on $\varepsilon$ a separated uniform structure, which we shall call the Hausdorff uniform structure on $\varepsilon$, following J. L. Kelley [5]. In this case, it is no longer true that the completeness of $E$ implies that of $\varepsilon$ (although, if $E$ is compact, $\varepsilon$ is then complete; see e.g. Bourbaki [1, Chapter II, §4, ex. 6]). An example of a complete uniform space $E$ for which $\varepsilon$ is not complete is provided by taking for $E$ any complete but not fully complete locally convex topological vector space, for the completeness of $\varepsilon$ implies the full completeness of $E$ (see [5]). The first result of this paper shows that the subset of $\varepsilon$ consisting of all the (nonempty) compact sets in $E$ is always complete, provided that $E$ is complete. The second theorem gives an extension of this result, which, applied to a complete locally convex topological vector space, shows that the weakly compact sets also form a complete space. The third theorem is concerned with function spaces. The corresponding result for a space of linear mappings can be regarded as a generalization of the fact, that, for Banach spaces, the limit of a convergent sequence of weakly compact linear mappings is weakly compact. This is because convergence in norm of a sequence of linear mappings is precisely convergence, in the Hausdorff uniform structure on the range space, of the sequence of closed images of the unit ball.

We have pleasure in thanking Dr. E. Michael for suggesting the generalization to uniform spaces of our results, originally stated for locally convex spaces, and for many helpful comments towards its achievement.

Let $E$ be a separated uniform space and $u$ a base for the uniform structure on $E$. If $U \in \mathcal{U}$ and $A \subseteq E$, we write $U(A)$ for the set of points $y$ with $(x, y) \in U$ for some $x \in A$. On $\mathcal{E}$, the set of closed nonempty subsets of $E$, the sets

Presented to the Society, January 28, 1960 under the title $O n$ the space of subsets of a topological vector space; received by the editors April 5, 1960 and, in revised form, May 23, 1960.

1 This paper was written while the authors were working under Contracts Nonr 58307 of the Office of Naval Research and G-4917 of the National Science Foundation. 


$$
W_{U}=\{(A, B): A \subseteq U(B) \text { and } B \subseteq U(A)\}, \quad U \in \mathcal{U},
$$

form a base for a separated uniform structure, called the Hausdorff uniform structure.

We collect some general facts about this structure. The net $\left(B_{\alpha}\right)$ in $\mathcal{E}$ is Cauchy if and only if to each $U \in \mathcal{U}$ corresponds $\alpha(U)$ with $B_{\alpha}$ $\subseteq U\left(B_{\beta}\right)$ for all $\alpha, \beta \geqq \alpha(U)$; it converges to $B$ if and only if there exists $\alpha(U)$ with $\mathrm{N} \subseteq U\left(B_{\alpha}\right)$ and $B_{\alpha} \subseteq U(B)$ for all $\alpha \geqq \alpha(U)$. If $t$ is a uniformly continuous mapping of $E$ into another separated uniform space $F$, the mapping $A \rightarrow \overline{t(A)}$ of $\mathcal{E}$ into the set $\mathcal{F}$ of closed nonempty subsets of $F$ is uniformly continuous for the Hausdorff uniform structures on $\mathcal{E}$ and F. Hence if $\left(B_{\alpha}\right)$ is Cauchy, so is $\left(\overline{t\left(B_{\alpha}\right)}\right)$ and if $B_{\alpha} \rightarrow B$ then $\overline{t\left(B_{\alpha}\right)}$ $\rightarrow \overline{t(B)}$. If $F$ is a closed subspace of $E$ then $\mathcal{F}$ is a closed subspace of $\varepsilon$. (For if $B \in \bar{F}$ and $U \in \mathcal{U}$, then $B \subseteq U(A)$ for some $A \in \mathcal{F}$; hence $B \subseteq U(F)$ for all $U \in \mathcal{U}$ and so $B \subseteq \bar{F}=F$.) Any increasing net $\left(B_{\alpha}\right)$ of sets whose union $B$ is precompact converges to $\bar{B}$. Any decreasing net which converges must converge to the intersection; so also does any Cauchy decreasing net of compact sets. If $B_{\alpha} \rightarrow B$ and each $B_{\alpha}$ is precompact, then so is $B$. Thus, in a complete space, if $B_{\alpha}$ converges to the (closed) set $B$ and each $B_{\alpha}$ is compact, then so is $B$.

TheOREM 1. Let $E$ be a complete separated uniform space and $\&$ the space of closed nonempty subsets of $E$ under the Hausdorff uniform structure. Then the nonempty compact subsets of $E$ form a complete subspace of $\varepsilon$.

Proof. Since $E$ is a separated uniform space, it can be embedded in a product $F=X\left\{E_{\gamma}: \gamma \in \Gamma\right\}$ of complete metric spaces (see e.g. Kelley $[4$, Chapter $6, \S 16])$. Let $\left(B_{\alpha}\right)$ be a Cauchy net of compact subsets of $E$. For each finite subset $\phi$ of $\Gamma$ let $p_{\phi}$ be the natural projection mapping of $F$ onto $F_{\phi}=\times\left\{E_{\gamma}: \gamma \in \phi\right\}$. Since $p_{\phi}$ is uniformly continuous, $\left(p_{\phi}\left(B_{\alpha}\right)\right)$ is a Cauchy net of compact subsets of the complete metric space $F_{\phi}$. Hence it converges, to the compact set $A_{\phi}$, say. In the special case when $\phi=\{\gamma\}$, we shall write simply $A_{\gamma}$. Put $A=X\left\{A_{\gamma}: \gamma \in \Gamma\right\}$ and, for each $\phi, C_{\phi}=A \cap p_{\phi}^{-1}\left(A_{\phi}\right)$. Then each $C_{\phi}$ is compact, being a closed subset of a product of compact sets; we prove that $C_{\phi}$ decreases as $\phi$ increases. For let $\psi \subseteq \phi$ and denote by $q$ the natural projection of $F_{\phi}$ onto $F_{\psi}$. Then clearly $q \circ p_{\phi}=p_{\psi}$, so that $q\left(p_{\phi}\left(B_{\alpha}\right)\right)=p_{\psi}\left(B_{\alpha}\right)$ for all $\alpha$, and therefore $q\left(A_{\phi}\right)=A_{\psi}$. Thus

$$
p_{\psi}\left(p_{\phi}^{-1}\left(A_{\phi}\right)\right)=\left(q \circ p_{\phi} \circ p_{\phi}^{-1}\right)\left(A_{\phi}\right)=q\left(A_{\phi}\right)=A_{\psi} \text { and so } C_{\phi} \subseteq C_{\psi} \text {. }
$$

In particular $C_{\phi} \subseteq C_{\gamma}$ for each $\gamma \in \phi$, so that $C_{\phi}=\times\left\{A_{\gamma}: \gamma \notin \phi\right\} \times A_{\phi}$, which proves that each $C_{\phi}$ is nonempty. Since the sets $C_{\phi}$ are compact 
and decrease with increasing $\phi$, their intersection $C$ is nonempty and compact.

Next, $p_{\psi}(C)=A_{\psi}$. For if $\psi \subseteq \phi, p_{\psi}\left(C_{\phi}\right)=q\left(A_{\phi}\right)=A_{\psi}$; hence, if $x \in A_{\psi}$, the sets $C_{\phi} \cap p_{\psi}^{-1}(x)$, with $\psi \subseteq \phi$, are nonempty. They are also compact and decrease as $\phi$ increases, and so their intersection $C \cap p_{\psi}^{-1}(x)$ is nonempty. Thus $A_{\psi} \subseteq p_{\psi}(C)$. But $p_{\psi}(C) \subseteq p_{\psi}\left(C_{\psi}\right)=A_{\psi}$.

Finally $B_{\alpha} \rightarrow C$. For a base of the product uniform structure on $F$ is formed by the sets $\left\{(x, y):\left(p_{\phi}(x), p_{\phi}(y)\right) \in U_{\phi}\right\}$ as $U_{\phi}$ runs through a base for the uniform structure on $F_{\phi}$ and $\phi$ runs through the finite subsets of $\Gamma$. Hence a base for the Hausdorff uniform structure on the set of closed nonempty subsets of $F$ is formed by the sets

$$
\left\{(A, B): p_{\phi}(A) \subseteq U_{\phi}\left(p_{\phi}(B)\right) \text { and } p_{\phi}(B) \subseteq U_{\phi}\left(p_{\phi}(A)\right)\right\} .
$$

Thus $B_{\alpha} \rightarrow C$ if and only if $p_{\phi}\left(B_{\alpha}\right) \rightarrow p_{\phi}(C)$ in $F_{\phi}$ for each finite $\phi \subseteq \Gamma$. But $p_{\phi}\left(B_{\alpha}\right) \rightarrow A_{\phi}=p_{\phi}(C)$ and so $B_{\alpha} \rightarrow C$.

Since $E$ is given to be complete, it is a closed subspace of $F$; each $B_{\alpha}$ lies in $E$ and therefore so does $C$. Thus the theorem is proved.

Given a uniform space $E$ with uniform structure $\xi$, we shall say that another uniform structure $\eta$ on $E$ is associated with $\xi$ if $\xi$ has a base consisting of subsets of $E \times E$ closed in the topology determined by $\eta$. This condition insures that $\xi$ can be extended to the $\eta$-completion $F$ of $E$. For if $\mathcal{U}$ is such a base for $\xi$, the $\eta$-closures in $F \times F$ of the sets of $\mathcal{u}$ form a base for a uniform structure on $F$ which induces $\xi$ on $E$. For simplicity, we shall continue to use $\xi$ and $\eta$ for the extensions of these structures to $F$.

Theorem 2. Let $E$ be a complete separated uniform space with uniform structure $\xi$ and let $\eta$ be a coarser separated uniform structure on $E$ associated with $\xi$. Then the set of nonempty $\eta$-compact subsets of $E$ is complete under the Hausdorff uniform structure corresponding to $\xi$.

Proof. We may suppose $\xi$ and $\eta$ extended to the $\eta$-completion $F$ of $E$. Let $\xi$ and $\tilde{\eta}$ be the corresponding Hausdorff uniform structures on the set $\mathfrak{C}$ of $\eta$-compact (and therefore also $\xi$-closed) subsets of $F$. If $\left(B_{\alpha}\right)$ is a $\xi$-Cauchy net of $\eta$-compact subsets of $E,\left(B_{\alpha}\right)$ is also Cauchy in $\mathfrak{e}$ under the coarser structure $\tilde{\eta}$ and so, by Theorem 1 , is $\tilde{\eta}$-convergent to some $\eta$-compact subset $B$ of $F$. Now let $\mathfrak{u}$ and $v$ be bases for $\xi$ and $\eta$ respectively, with the sets of $u$-closed. If $U \in \mathcal{U}$ is given, there is an $\alpha_{0}$ with $B_{\alpha} \subseteq U\left(B_{\beta}\right)$ for all $\alpha, \beta \geqq \alpha_{0}$. Then for each $V \in \mathcal{V}$ there is an $\alpha(V) \geqq \alpha_{0}$ with $B_{\alpha} \subseteq V(B)$ and $B \subseteq V\left(B_{\alpha}\right)$ for all $\alpha \geqq \alpha(V)$. Thus, if $\beta \geqq \alpha_{0}, B \subseteq V\left(B_{\alpha}\right) \subseteq V\left(U\left(B_{\beta}\right)\right)$ for a suitable $\alpha$. Hence

$$
B \subseteq \cap\left\{V\left(U\left(B_{\beta}\right)\right): V \in v\right\}, \text { and similarly }
$$




$$
B_{\beta} \subseteq \cap\{U(V(B)): V \in v\} .
$$

If we show that this implies that $B \subseteq U\left(B_{\beta}\right)$ and $B_{\beta} \subseteq U(B)$ we shall have proved that $\left(B_{\alpha}\right)$ converges to $B$ in $\xi$; since $E$ is a $\xi$-closed subset of $F$, it will then follow that $B \subseteq E$ and the theorem will be proved. It will be sufficient to show that, if $A$ is $\eta$-compact,

$$
\cap\{V(U(V(A))): V \in v\} \subseteq U(A) .
$$

If $y$ belongs to the left side of this, there are points $x_{V} \in A$ with $\left(x_{V}, y\right) \in V U V$. Let $x$ be an $\eta$-adherent point of $\left(x_{V}: V \in V\right)$; then $x \in A$ and to each $V$ corresponds a $V^{\prime} \subseteq V$ with $\left(x, x_{V^{\prime}}\right) \in V$. Thus $(x, y) \in V^{\prime} U V^{\prime} V \subseteq V V U V V$ for all $V \in V$. Since $U$ is $\eta$-closed, $(x, y)$ $\in U$ and $y \in U(A)$, as required.

CoRollary. In the space of nonempty $\xi$-closed subsets of $E$ with the Hausdorff uniform structure corresponding to $\xi$, the $\eta$-relatively compact subsets of $E$ form a closed subspace.

Proof. Suppose that each $B_{\alpha}$ is $\eta$-relatively compact and that $B_{\alpha} \rightarrow B$ under $\xi$. If $U \in \mathcal{U}$, there is an $\alpha_{0}$ such that, for all $\alpha, \beta \geqq \alpha_{0}$, $B_{\alpha} \subseteq U\left(B_{\beta}\right)$. Hence, if bars denote $\eta$-closures, $\overline{B_{\alpha}} \subseteq V\left(U\left(B_{\beta}\right)\right)$ $\subseteq V\left(U\left(\overline{B_{\beta}}\right)\right)$ for all $V \in \mathcal{V}$. Because $\overline{B_{\beta}}$ is $\eta$-compact, it follows as in the theorem that $\overline{B_{\alpha}} \subseteq U\left(\overline{B_{\beta}}\right)$, so that $\left(\overline{B_{\alpha}}\right)$ is $\tilde{\xi}$-Cauchy. Hence $\left(\overline{B_{\alpha}}\right)$ is $\xi$-convergent to the $\eta$-compact set $C$, say. Now, if $V \in \mathcal{V}$, we have, for sufficiently large $\alpha$, since $\xi$ is finer than $\eta$,

$$
B \subseteq V\left(B_{\alpha}\right) \subseteq V\left(\overline{B_{\alpha}}\right) \subseteq V(V(C)) .
$$

Hence $B \subseteq C$ and $B$ is $\eta$-relatively compact.

The space of $\eta$-relatively compact subsets of $E$ need not be complete; there is a counterexample later. The corollary has an immediate application to function spaces.

Theorem 3. Let $S$ be a set and a a family of subsets. Also let $E$ be a complete separated uniform space with uniform structure $\xi$ and let $\eta$ be a coarser separated uniform structure on $E$ associated with $\xi$. If $F$ is the space of mappings of $S$ into $E$ which take the sets of a onto $\eta$-relatively compact subsets of $E$, then $F$ is complete under the uniform structure of $\xi$-uniform convergence on the sets of $a$.

Proof. Let $\left(f_{\alpha}\right)$ be a Cauchy net in $F$. Since the space $E^{S}$ of all mappings of $S$ into $E$ is complete under the $(a, \xi)$ uniform structure, $\left(f_{\alpha}\right)$ converges in $E^{s}$ to $f$, say. It follows that, for each $A \in Q$, the $\xi$-closure of $f_{\alpha}(A)$ converges to the $\xi$-closure of $f(A)$ under the Hausdorff uniform structure corresponding to $\xi$. Since each $f_{\alpha}(A)$ is $\eta$ relatively compact, the corollary of Theorem 2 shows that $f(A)$ is 
also $\eta$-relatively compact. Hence $f \in F$ and $F$ is complete.

The motive for considering associated uniform structures comes from the theory of locally convex spaces. Here we may speak about topologies instead of uniform structures. Let $\xi$ and $\eta$ be two separated locally convex topologies on a vector space $E$. Then (the uniform structure determined by) $\eta$ is associated with (that determined by) $\xi$ if and only if there is a base of $\eta$-closed $\xi$-neighbourhoods of the origin. An immediate example, the one which suggested the terminology, is obtained by taking for $\eta$ the associated weak topology corresponding to $\xi$. The two topologies $\xi$ and $\eta$ are each associated with the other if and only if there is a vector space $F$ is duality with $E$ such that each of $\xi$ and $\eta$ is a topology of uniform convergence on a family of $\sigma(F, E)$-bounded subsets whose union spans $F$. (For if this last condition is satisfied there is a base of $\xi$-neighbourhoods of the origin consisting of polars of subsets of $F$; they are therefore $\sigma(E, F)$-closed and so closed in the finer topology $\eta$. Conversely, if $\xi$ and $\eta$ are associated, we can take for $F$ the intersection of the duals $E_{\xi}^{\prime}$ and $E_{\eta}^{\prime}$. If $U$ is an $\eta$-closed $\xi$-neighbourhood of the origin, which we may suppose absolutely convex, its polar in $E_{\eta}^{\prime}$ is $U^{\circ} \cap F$ and so the polar of $U^{\circ} \cap F$ in $E$ is the $\eta$-closure of $U$, which is $U$ itself. Thus $\xi$ is the topology of uniform convergence on sets of the form $U^{\circ} \cap F$, and similarly for $\eta$.)

The natural analogue of Theorem 3 for linear spaces is the following, obtained at once from Theorem 3 . Let $S$ be a separated locally convex space, and $E$ a complete separated locally convex space with topology $\xi$. Also let $\eta$ be a coarser separated topology on $E$ associated with $\xi$. Then the space of linear mappings of $S$ into $E$ which take bounded sets of $S$ onto $\eta$-relatively compact subsets of $E$ is complete under the topology of $\xi$-uniform convergence on the bounded subsets of $S$.

We make one or two additional comments on Theorem 2, when $E$ is a locally convex space. It is immediate that, with the notations used in the theorem, every $\tilde{\eta}$-complete set of $\eta$-compact sets is also $\tilde{\xi}$-complete. A proof that closely parallels that of Theorem 2 shows that, if $E$ has the same dual under $\xi$ and $\eta$, every $\tilde{\eta}$-complete set of closed convex subsets of $E$ is also $\xi$-complete. (Compare the corresponding result for points: Bourbaki [2, Chapter I, \$1, Proposition 8].)

In a complete separated locally convex space, although the weakly compact subsets form a complete space, the space of closed bounded subsets may fail to be complete. We shall give an example of a nonconvergent Cauchy net of closed bounded subsets of a product $E$ of uncountably many copies of the Banach space $m$. This will also furnish the counterexample promised after the corollary to Theorem 2. For if $\xi$ is the product of the norm topologies and $\eta$ 
the (associated) product of the topologies $\sigma\left(m, l^{1}\right)$, the sets of the net will be $\eta$-relatively compact, since each bounded set in $m$ is $\sigma\left(m, l^{1}\right)$-relatively compact.

Let $\Gamma$ be an uncountable set of indices and put $E=X\left\{E_{\gamma}: \gamma \in \Gamma\right\}$, where, for each $\gamma \in \Gamma, E_{\gamma}$ is the Banach space $m$. On $E$, we consider the product of the norm topologies. We shall say that a subset $A$ of $m$ has the property $\left({ }^{*}\right)$ if every element of $A$ is a sequence of nonnegative numbers converging to zero and the sum $s_{B}$ of the elements in each (nonempty) finite subset $B$ of $A$ has norm in the range $3 / 4$ $\leqq\left\|s_{B}\right\| \leqq 1$. There are such sets; if $e_{n}$ denotes the sequence whose $n$th term is 1 and all of whose other terms are 0 , any finite or infinite subset of $\left\{e_{n}: n=1,2, \cdots\right\}$ has the property $\left({ }^{*}\right)$. But if $A$ has the property $\left({ }^{*}\right)$, then $A$ is finite or countable, because, for each $n$, there is at most one sequence in $A$ whose $n$th term is greater than or equal to 3/4, and each sequence in $A$ has at least one such term. Now, for each finite subset $\phi$ of $\Gamma$ let $B_{\phi}$ be the subset of $E$ consisting of those points $x=\left(x_{\gamma}\right)$ for which $\left\{x_{\gamma}: \gamma \in \phi\right\}$, regarded as a subset of $m$, has the property $\left({ }^{*}\right)$, and $\left\|x_{\gamma}\right\| \leqq 1$ for all $\gamma \in \Gamma$. Then each $B_{\phi}$ is bounded in $E$, and is closed, being an intersection of closed sets. We show now that, if $p_{\phi}$ is the natural projection of $E$ onto $\times\left\{E_{\gamma}: \gamma \in \phi\right\}, p_{\phi}\left(B_{\psi}\right)$ $=p_{\phi}\left(B_{\phi}\right)$ for all $\phi \subseteq \psi$. Since the sets $B_{\psi}$ decrease with increasing $\psi$ we need only prove that if $\left\{x_{\gamma}: \gamma \in \phi\right\}$ has the property $\left({ }^{*}\right)$, points $\left\{x_{\gamma}: \gamma \notin \phi\right\}$ can be so defined that $\left(x_{\gamma}: \gamma \in \Gamma\right) \in B_{\psi}$. If $\phi$ has $r$ elements, we can choose $k$ so large that the $n$th coordinate of $x_{\gamma}$ is less than $(4 r)^{-1}$ for all $n \geqq k$ and all $\gamma \in \phi$. Then for each $\gamma$ in $\psi$ but not in $\phi$ we choose distinct integers $n(\gamma) \geqq k$ and put $x_{\gamma}=(3 / 4) e_{n(\gamma)}$. Finally for $\gamma \notin \psi$ we put $x_{\gamma}=o$. It is then a routine matter to check that this definition will suffice. Because $p_{\phi}\left(B_{\psi}\right)$ is constant for all $\psi$ with $\phi \subseteq \psi,\left(B_{\psi}\right)$ is a Cauchy net. Also, since $\left(B_{\psi}\right)$ is decreasing, if it converges, it must converge to the intersection of the sets $B_{\psi}$. But this intersection is empty, for if $x=\left(x_{\gamma}\right)$ were a point in it, the uncountable set $\left\{x_{\gamma}: \gamma \in \Gamma\right\}$ would have the property $\left({ }^{*}\right)$. Hence $\left(B_{\psi}\right)$ does not converge.

\section{REFERENCES}

1. N. Bourbaki, Topologie genérale, Paris, Hermann, 1940-1949.

2. - Espaces vectoriels topologiques, Paris, Hermann, 1953-1955.

3. F. Hausdorff, Mengenlehre, 2d ed., Berlin, Gruyter, 1927.

4. J. L. Kelley, General topology, New York, Van Nostrand, 1955.

5. - Hypercomplete linear topological spaces, Michigan Math. J. vol. 5 (1958) pp. 235-246.

6. C. Kuratowski, Topologie. I, Monografje Matematyczne, vol. 3, Warsaw, 1933.

7. G. B. Price, On the completeness of a certain metric space, Bull. Amer. Math. Soc. vol. 46 (1940) pp. 278-280.

UNIVERSITY OF KANSAS 